\title{
PLASMA EDGE DIAGNOSTICS FOR TEXTOR
}

\author{
S. BREZINSEK and A. HUBER Institut für Plasmaphysik, Forschungszentrum Jülich GmbH \\ EURATOM Association, Trilateral Euregio Cluster, D-52425 Jülich, Germany
}

S. JACHMICH Laboratory for Plasma Physics, Ecole Royale Militaire - Koninklijke Militaire School Association EURATOM-Belgian State, Trilateral Euregio Cluster, B-1000 Brussels, Belgium

A. POSPIESZCZYK,* B. SCHWEER, and G. SERGIENKO Institut für Plasmaphysik

Forschungszentrum Jülich GmbH, EURATOM Association, Trilateral Euregio Cluster, D-52425 Jülich, Germany

Received June 4, 2004

Accepted for Publication September 11, 2004

The exploration of plasma-wall-interaction physics is one of the major tasks of the tokamak TEXTOR. A characterization of the high-temperature plasma edge is essential to interpret the interaction processes of the different charged and uncharged particles in the boundary layer. In the design of the TEXTOR, much effort was made to optimize diagnostic access to the plasma edge for the best possible characterization. The major part of the plasma edge diagnostics presented here is based on passive and active spectroscopy, in addition to different types of electrical probes. Thereby, pioneering work has been achieved in both fields.

In passive emission spectroscopy, the work concentrated on the determination of particle fluxes of different types of atomic $(W, S i, C, \ldots)$ and molecular $\left(D_{2}, C D\right.$, $\mathrm{C}_{2}, \ldots$. species from the corresponding photon fluxes at different locations and on the visualization of the local impurity sources by means of two-dimensional imaging. The active spectroscopy with atomic beams was focused on the determination of plasma edge parameters $\left(n_{e}, T_{e}\right.$, $T_{i}, \ldots$. ) with good spatial and temporal resolution. Therefore, different techniques like thermal Li and He beams, suprathermal Li beams-realized by laser blow-off techniques-and hydrogen neutral beam injectors have been employed. Furthermore, laser-induced fluorescence measurements in the ultraviolet and in the vacuum ultraviolet ranges, which were for the first time performed in a fusion plasma, are presented. The continuous improvement of the different plasma edge diagnostics over more than a decade of TEXTOR plasma operation with different types of first-wall materials is discussed.

KEYWORDS: plasma diagnostics, spectroscopy, plasmawall interaction

\section{INTRODUCTION}

The edge of a fusion plasma is characterized by an interplay of various physical processes. There the neutral and charged particles, which move freely or along magnetic field lines, respectively, come into contact with solid objects like limiters, divertor plates, and the wall. At this point they can release other particles, return to the plasma in the same form or as molecular compounds, stick to the surface, or migrate into the solid. Therefore, plasma physics, surface science, and solid-state physics are strongly involved in all these processes and interact with each other.

*E-mail: A.Pospieszczyk@fz-juelich.de
One major topic deals with the particle release from the objects mentioned above as this influences the purity and the particle content. The more nonhydrogen particles can enter the plasma, the more energy will be radiated and gets lost; in addition, they will dilute the fuel and reduce the fusion energy gain. Moreover, if the fuel is badly confined, more energy has to be used for the ionization of the additionally penetrating particles. Therefore, it is important to provide answers to the following questions. How many particles of which kind are released, and where do they move and how fast? Into which kind of plasma do they enter, and what are the interactions of such a plasma with them?

Unfortunately, the possibilities to investigate these properties are rather limited in the case of a toroidally 


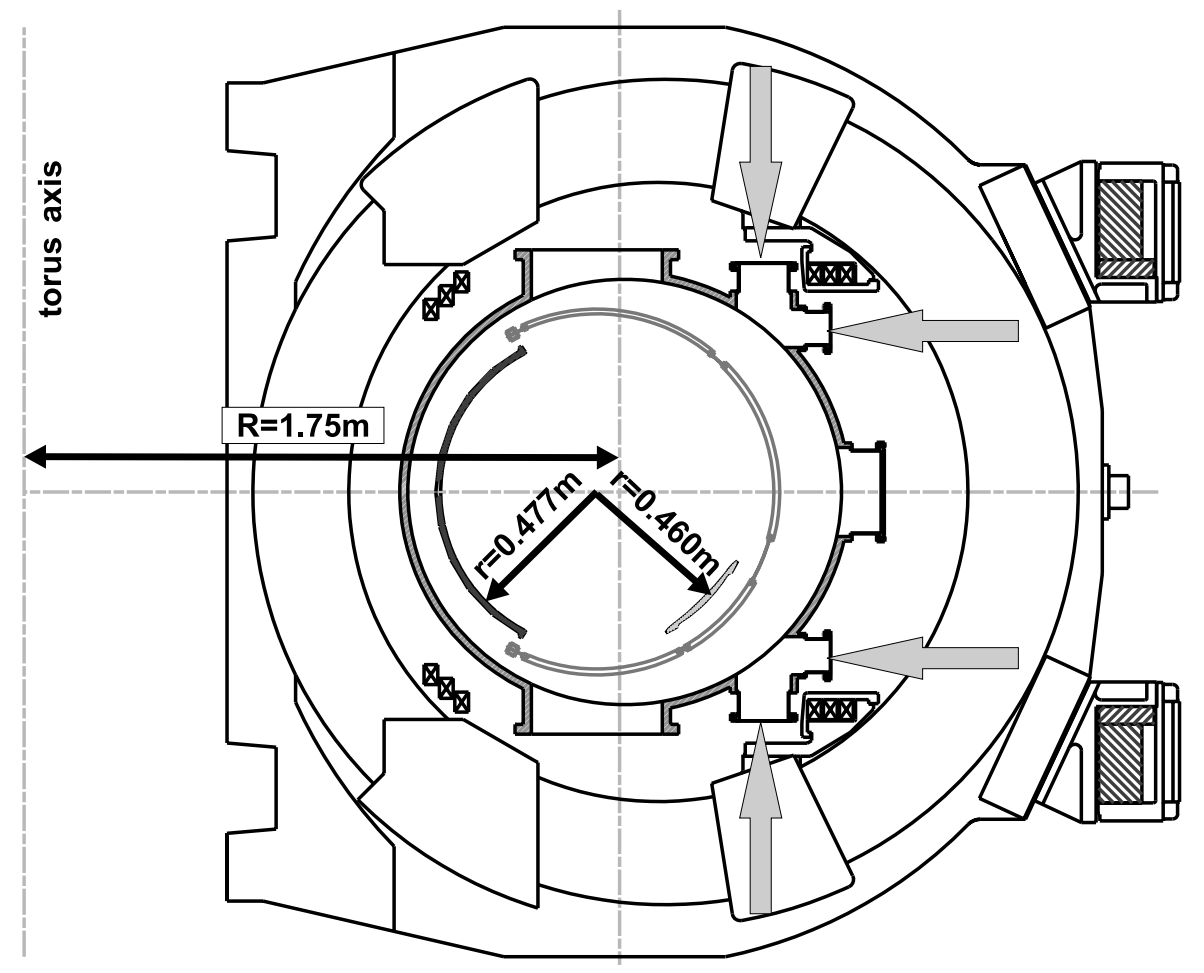

Fig. 1. Representative poloidal cross section of TEXTOR with two horizontal and two vertical observation lines, all indicated by arrows, for the plasma edge.

shaped fusion plasma experiment and its hostile surroundings for sophisticated diagnostic techniques. These should be robust and work reliably, should not disturb the plasma or alter it, and should be able to determine all these quantities mentioned for a variety of particles and plasma conditions. Since the first days of TEXTOR operation, spectroscopic methods and after a short while electrical probes have been employed for these purposes. Whereas probe techniques may suffer from too-strong interaction with the plasma, the spectroscopic methods do not touch objects and have, in most cases, proven their reliability in the investigations of astrophysical plasmas. ${ }^{1}$ Moreover, in laboratories active techniques using lasers or lowcurrent particle beams can also be employed for nondisturbing measurements.

Another advantage of the use of spectroscopic methods is important. The fusion plasma edge is determined by both a wide range of variations in the plasma parameters and their steep gradients within a couple of centimeters. For an estimation one may regard the ionization length of hydrogen as a guide for its width in plasmas characterized by $10^{17} \mathrm{~m}^{-3}<n_{e}<10^{21} \mathrm{~m}^{-3}, 1 \mathrm{eV}<T_{e}<$ $100 \mathrm{eV}, 10^{-3} n_{e}<n_{I}<10^{-1} n_{e}, n_{\mathrm{H} 0} \approx 10^{-3} n_{e}$ with $n_{e}$ the electron density, $T_{e}$ the electron temperature, $n_{I}$ the impurity density, and $n_{\mathrm{H} 0}$ the atomic hydrogen density. The requirements for detecting changes within millimeters and over orders of magnitude can best be fulfilled by optical detection techniques. Therefore, during the planning of TEXTOR, much care was taken to design ports for easy optical access to the plasma boundary region. Figure 1 shows an example of how the access was accomplished by a number of tangential viewing lines. The radial access was then used for the application of photon or particle beams, which could be observed with high radial resolution.

\section{PASSIVE EMISSION SPECTROSCOPY}

Passive plasma boundary spectroscopy on TEXTOR has begun with the observation of light emission from the limiters and walls both by spectrometers, fast integrating diodes, and charge-coupled-device (CCD) cameras, which - in combination with interference filters (IFs) allow one to record two-dimensional intensity distributions of specific atoms and ions. Figure 2 shows a poloidal cross section taken in the light of $\mathrm{H}_{\alpha}$ with a CCD camera, in which the observation volumes for the detection systems are indicated. Whereas the fast diodes integrate the light emission from the whole volume via an appropriate IF, the spectrometer also yields radial intensity distributions (imaging). The latter can also be used to roughly determine the plasma boundary parameters in front of the limiters. $^{2}$ 


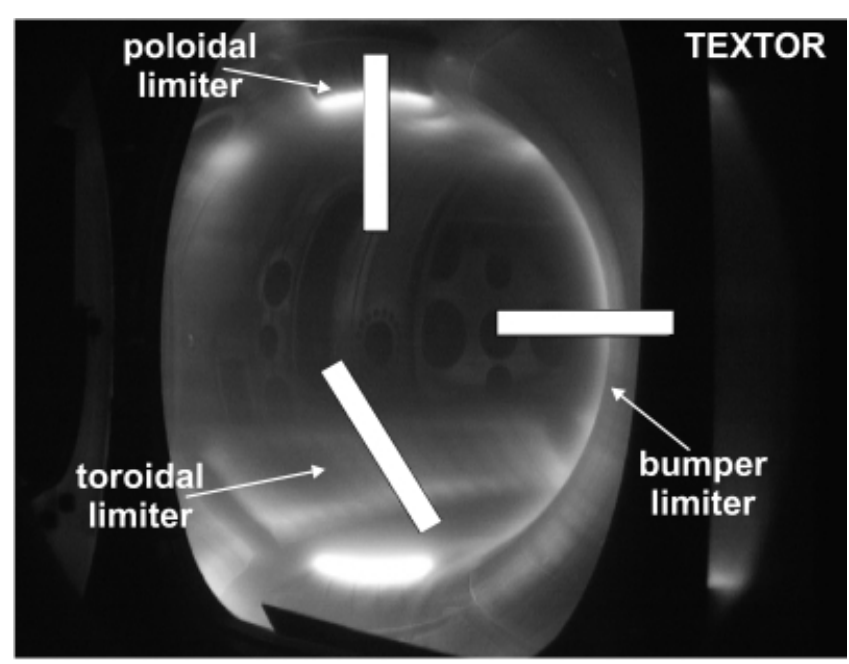

Fig. 2. TEXTOR discharge in the light of $\mathrm{H}_{\alpha}$. The areas denote the regions of integrated and spatially resolved spectroscopic measurements.

The integrated signal serves to determine particle fluxes in the case of an ionizing plasma, i.e., that all radiation from particles vanishes by entering the next ionization state. In this case the number of ionizations equals the number of particles penetrating into the plasma, i.e., the particle flux. The conversion of photon into particle fluxes is not always a trivial task and often requires profound knowledge of the population mechanism for the atomic levels with respective emission and ionization rates. The principles have been outlined in detail in Refs. 2 and 3.

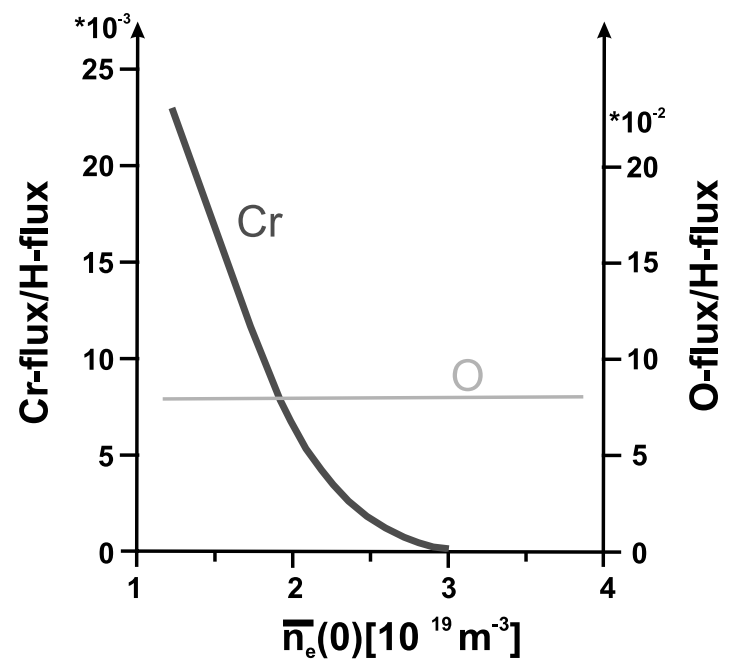

Fig. 3. Relative oxygen and chromium fluxes from the poloidal (main) limiter as a function of the line-averaged central density $n_{e}(0)$ in the "all metal" TEXTOR.

First results ${ }^{2}$ show a plasma, which is governed by relatively large oxygen and metal fluxes (Fig. 3) and characterized by a short pulse duration. Thereby, the short pulse duration was mainly caused by the radiation of medium-Z impurities in the plasma core. This led to the replacement of the metal (stainless steel) limiter by a graphite one accompanied by a carbonization technique, which resulted in a diamond-like carbon coating of the wall. The oxygen fluxes were strongly reduced (Fig. 4) and the pulse duration increased. ${ }^{4}$

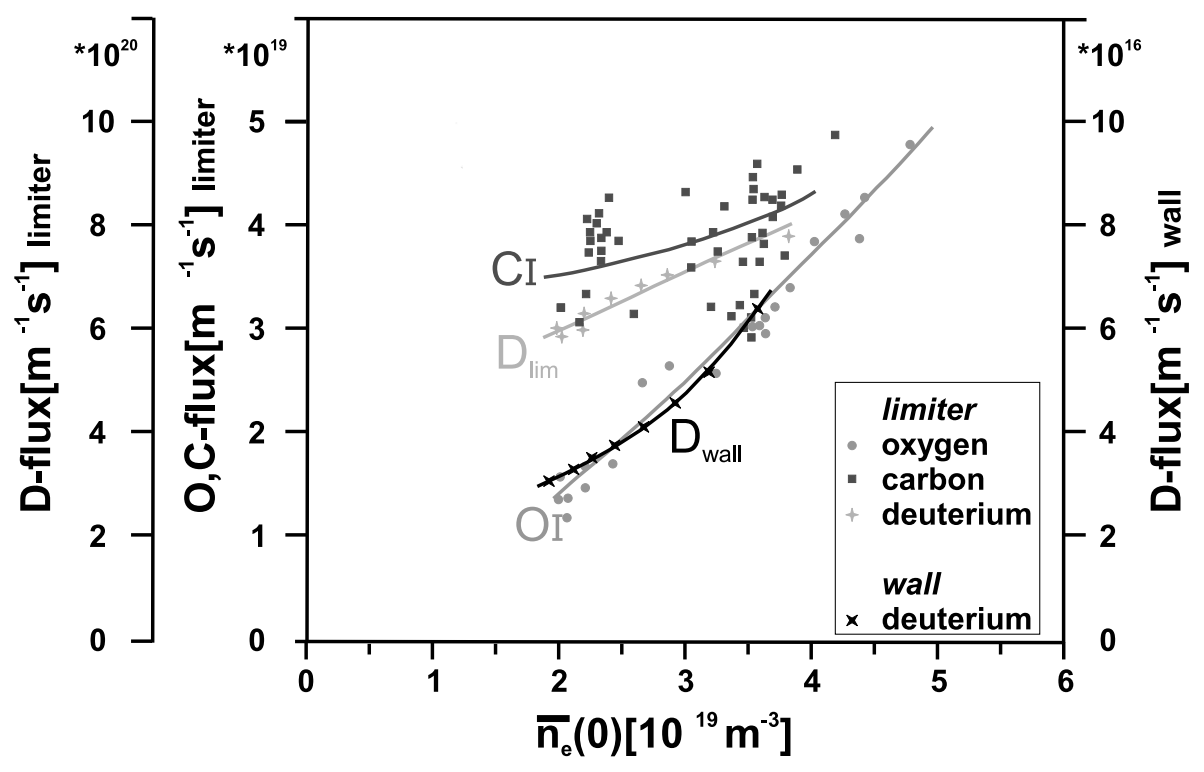

Fig. 4. Fluxes of several elements after carbonization. 


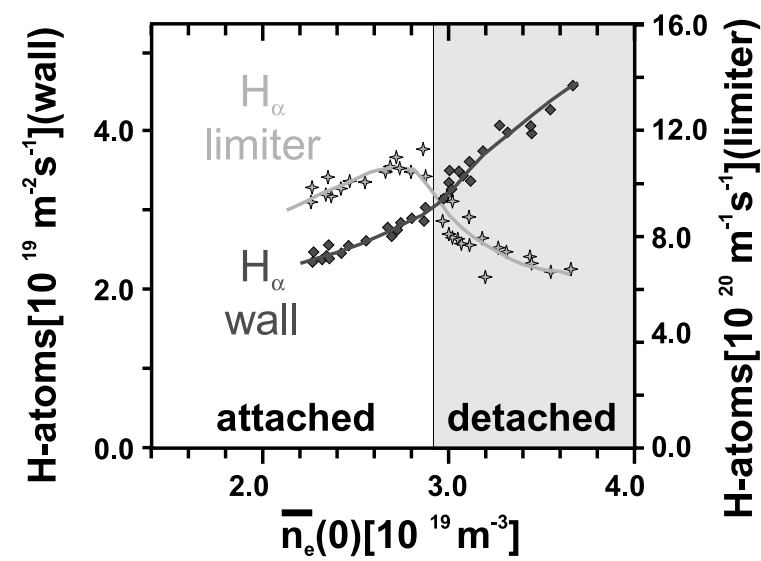

Fig. 5. Particle flux from limiter and wall after carbonization.
Also, the plasma entered into a new state, which was characterized by detachment from the limiters and increased contact with the wall ${ }^{5}$ (Fig. 5). The hydrogen fluxes to the limiters dropped, and a cold, recombining plasma layer was formed.

Another interesting feature, combined with the introduction of carbon into the plasma chamber, was the appearance of organic molecules (hydrocarbons) created by interaction of the working gas with the graphite surfaces. This introduced not only a completely new kind of spectroscopy into plasma boundary research, i.e., the spectroscopy of molecules with their numerous lines and band structures (Fig. 6), but also, with the chemical erosion new release mechanisms. Therefore, the edge spectroscopy was upgraded by highly resolving instruments, ${ }^{6}$

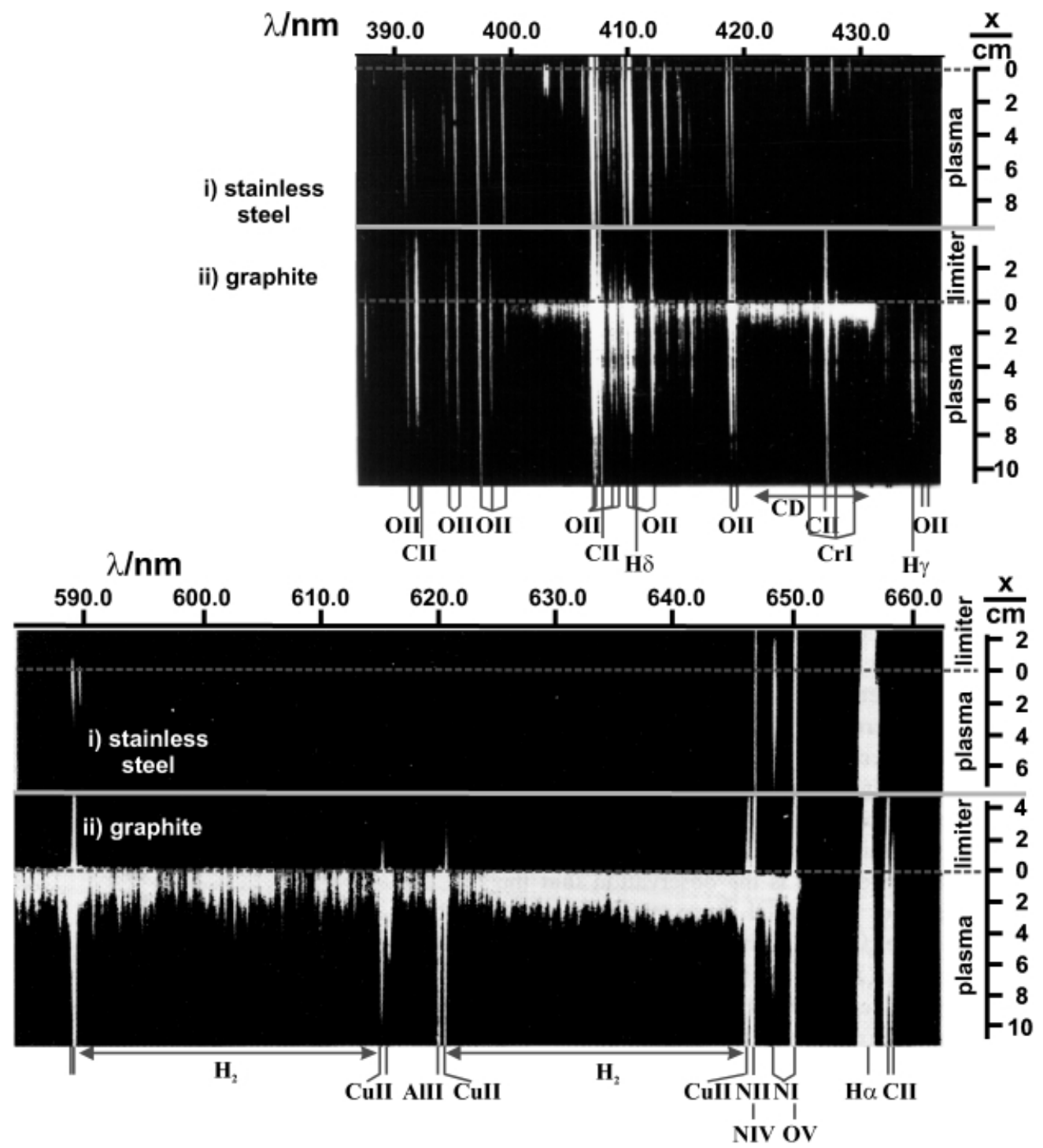

Fig. 6. Spectra of the main plasma limiter interaction zone for two types of poloidal limiters: (a) wavelength range $\sim 410 \mathrm{~nm}$ and (b) wavelength range $\sim 620 \mathrm{~nm}$. 


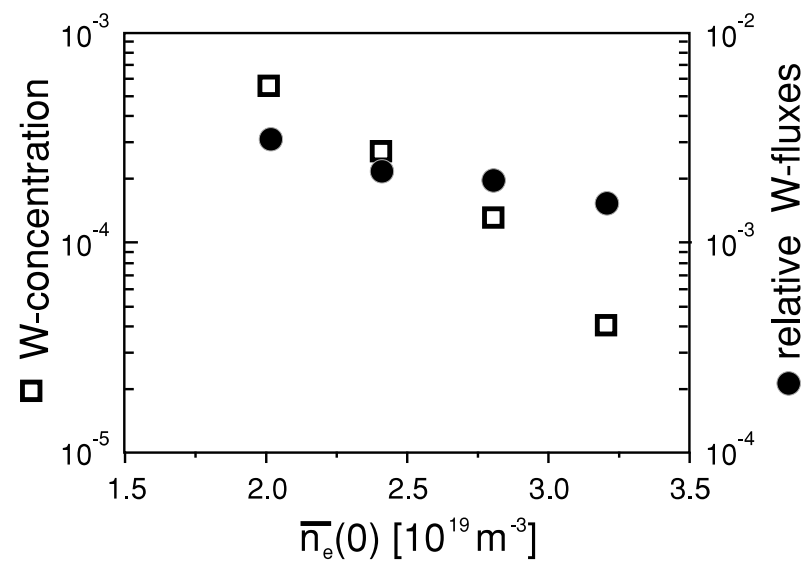

Fig. 7. Tungsten concentration and fluxes during a density scan with ICRH-power of $1 \mathrm{MW}$. The poloidal W limiter was located at the LCFS.

which could resolve the individual structures of hydrogen $^{7-9}$ hydrocarbons and molecular carbon. ${ }^{10}$

Another improvement of the imaging spectrometers was achieved by the replacement of the photographic recording by an intensified CCD camera as detector. An image processing unit now allowed fast quantitative evaluation of the penetration of the different species into the plasma, which yielded the screening efficiencies for several limiter and their released particles. It was found that even tungsten can be used as material (Fig. 7) provided that the penetration depth can be kept small by an appro-

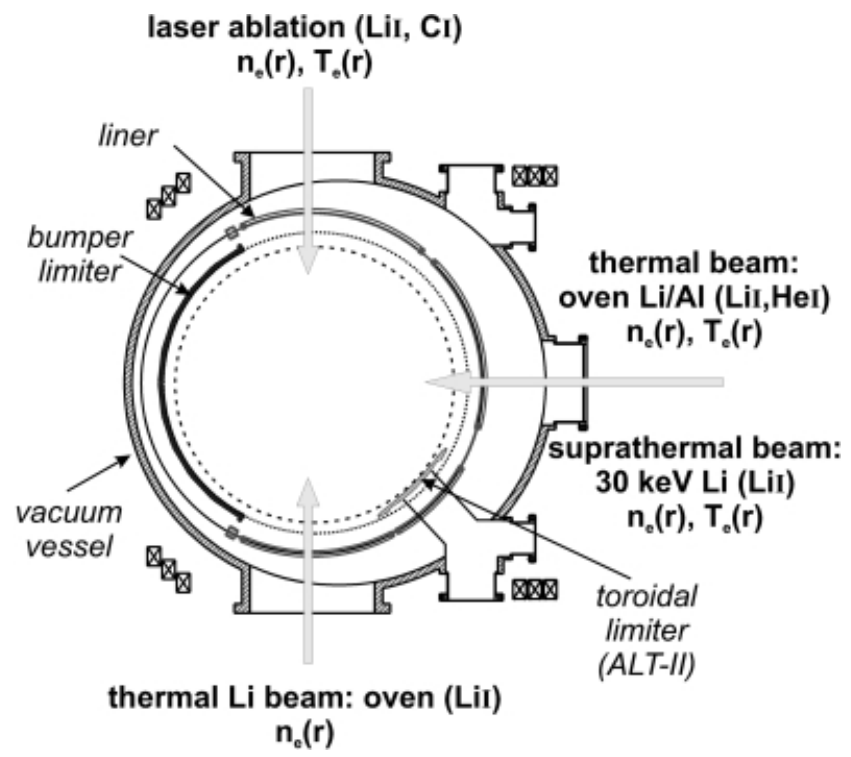

Fig. 8. Projection of diagnostics using atomic beams into one poloidal cross section showing their location in poloidal direction in TEXTOR.

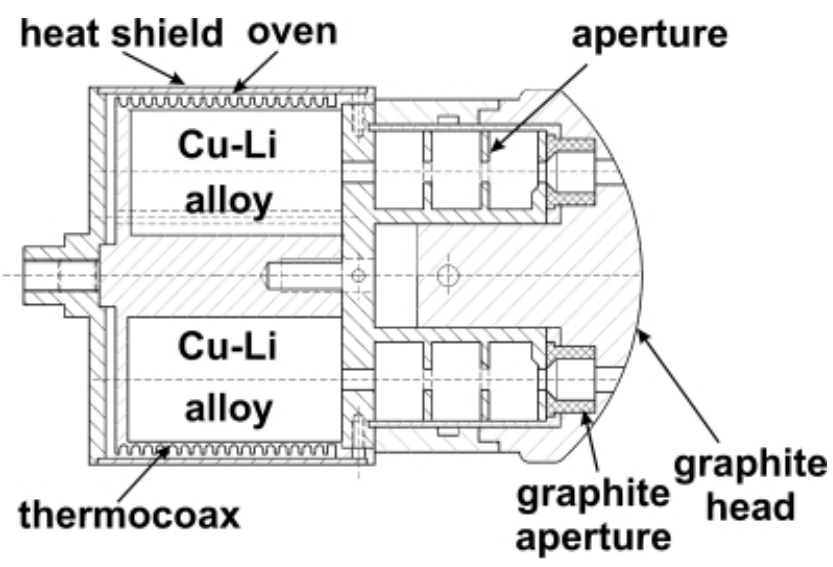

Fig. 9. Cut through a double-beam thermal Li oven used for fluctuation measurement of the electron density.

priate shaping of the boundary plasma. ${ }^{11}$ Recently, the video recording system, which worked reliably for $>15 \mathrm{yr}$, was exchanged for a fully computerized digital recording system, ${ }^{12}$ which now allows the storage of hundreds of gigabytes for a number of cameras for plasma spectroscopic purposes.

\section{DIAGNOSTICS WITH ATOMIC BEAMS}

The emission signals obtained by passive spectroscopy already contain much information about temperature, density, and flux of the main species and impurities. ${ }^{3}$ The interpretation of these measured line intensities requires a knowledge of atomic physics describing the specific radiation from the plasma. Tomographic methods are applied, but they need symmetries for the calculation

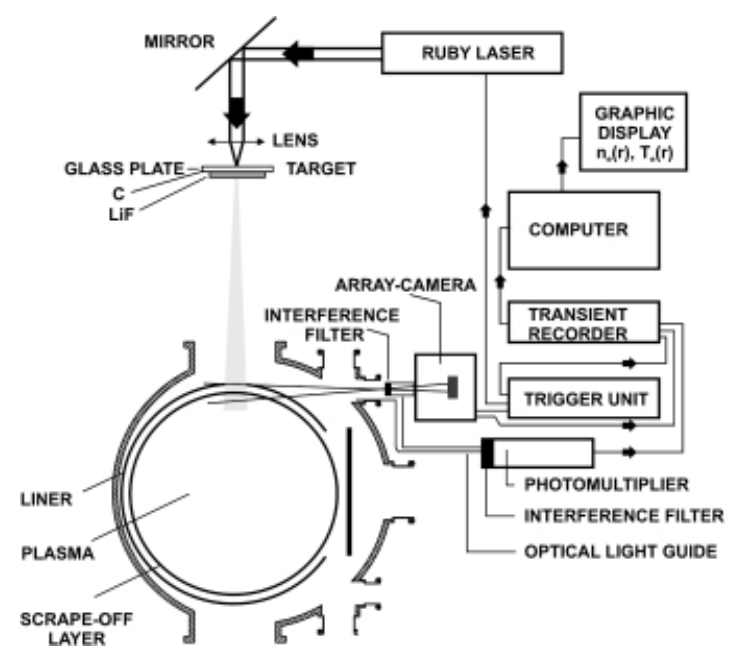

Fig. 10. Setup of the laser ablation system on TEXTOR. 


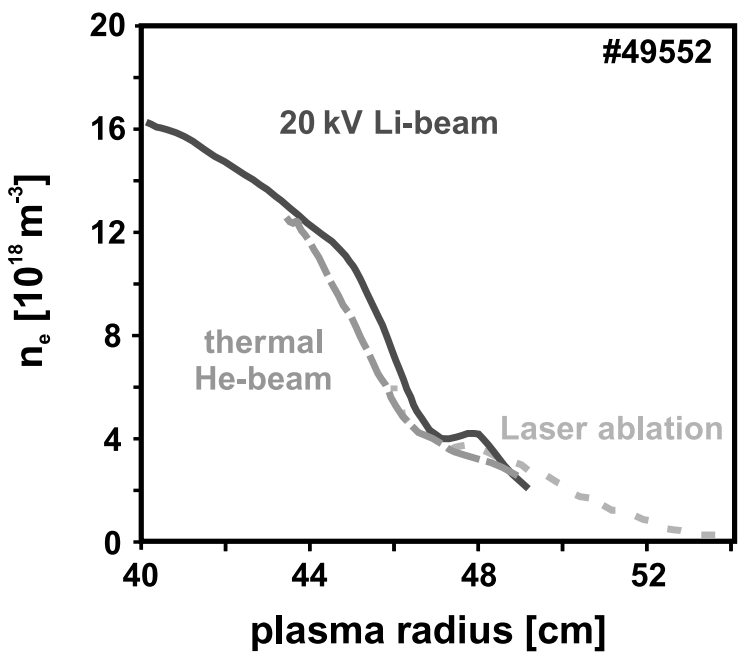

Fig. 11. Comparison of $n_{e}$ profiles obtained with different atomic beam diagnostics.

of local parameters. Also, asymmetries and steep gradients of plasma parameters that appear in the plasma boundary of a tokamak or stellarator require the direct local measurement of these quantities. Therefore, the local addition of atoms, which have known atomic properties, allows a well-resolved local determination of the plasma characteristics.

From its beginning many diagnostic ports on TEXTOR for the observation of the boundary layer have been designed in cross-beam geometry, i.e., a particle injection system was installed on one port and observed through a second one, which looked perpendicular onto the beam. Figure 8 shows such an arrangement for one poloidal cross section. Thus, a maximum of spatial resolution was obtained. If the beam was confined to a reasonably small size, the integration over the line of sight could be neglected. ${ }^{13}$

The pioneering work commenced with thermal particle sources. Such sources are easy to produce. Their energies are below $0.1 \mathrm{eV}$, and they can deliver a high continuous particle flux, which can be modulated by mechanical choppers. Their disadvantage is the large divergence that can be reduced only by the introduction of apertures into the beam. Clearly, thermal sources are the least costly and most reliable beams if solid-state emitters are used. They have a large inventory, and therefore, they can be operated over long periods of time. An example of this kind of source was a thermal lithium beam. Initially, pure lithium was introduced into an oven and heated to $600^{\circ} \mathrm{C}$. The Li flux (partial pressure $\approx 1 \mathrm{mbar}$ ) was so high that the source could be placed $\sim 1$ m distance from the plasma. But, the liquid Li was difficult to handle, and also, the formation of a solid $\mathrm{Li}_{2} \mathrm{O}$ and $\mathrm{LiH}_{2}$ layer reduced the reliability of the source. The use of

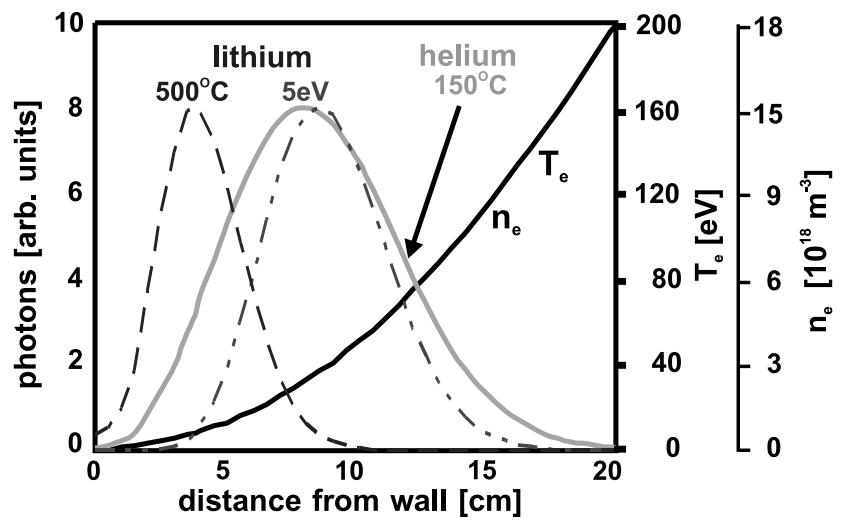

Fig. 12. Comparison of penetration depths for thermal $\mathrm{Li}$ (- - -), thermal He (_) and suprathermal Li (_.....).

$\mathrm{Al} / \mathrm{Li}$ (melting point $604^{\circ} \mathrm{C}$ ) or $\mathrm{Cu} / \mathrm{Li}$ (melting point $1000^{\circ} \mathrm{C}$ ) alloys, which have strong diffusion of $\mathrm{Li}$ atoms to the surface, ${ }^{14}$ allowed one to build reliable and mountable sources in any direction with long operational times $(>500 \mathrm{~h})$. A cut through such an oven used for fluctuation measurements with two beams is shown in Fig. 9.

At a distance $100 \mathrm{~mm}$ from the source, which is the typical distance to the measurement volume, $\mathrm{Cu} / \mathrm{Li}$ alloy at a temperature of $700^{\circ} \mathrm{C}$ delivers flux densities of $5 \times$ $10^{18} \mathrm{~m}^{-2} \cdot \mathrm{s}^{-1}$. The corresponding velocity of the $\mathrm{Li}$ atoms is $1.7 \mathrm{~km} / \mathrm{s}$ so that a density of $3 \times 10^{15} \mathrm{~m}^{-3}$ is possible. ${ }^{15}$

"Suprathermal" beams with higher energies up to 10 $\mathrm{eV}$, which allow probing up to $6 \mathrm{~cm}$ into the confined plasma region, are produced by the laser blow-off technique. Thin films (e.g., LiF) are deposited on glass or quartz substrates. A powerful laser beam is focused onto the rear side of the substrate. The material absorbs the radiation and forms an atom beam with a low divergence of $\pm 5 \mathrm{deg}$. However, this method allows only pulsed operation $(100 \mu \mathrm{s})$. The determination of the background light from the plasma is done before or after the cloud has passed the observation volume. Very high particle fluxes, pre-selected by the spot size, are achieved, but only condensing materials can be chosen. Lasers with repetition rates up to $30 \mathrm{~Hz}$ are applied. However, the powerful laser system and the target movement and control system are expensive. A sketch of how such a system was applied on TEXTOR (Refs. 15 and 16) for the injection of, for instance, $\mathrm{Li}$ and $\mathrm{Al}$, is shown in Fig. 10. Lithium was chosen in both systems because its radiation characteristics are independent of electron temperatures $>10 \mathrm{eV}$ and allowed, therefore, easy conversion of photon density profiles into electron density profiles.

Finally, a special solid-state source for $\mathrm{Li}$ ions was used in several experiments. ${ }^{17} \mathrm{~A} \beta$-eucryptite emitter is heated to $1300^{\circ} \mathrm{C}$. If voltage is applied $(20$ to $30 \mathrm{kV}$ ), surface ionization occurs, and $\mathrm{Li}$ ions are extracted, which are neutralized in $\mathrm{Li}$ or $\mathrm{Na}$ vapor. This source is reliable and can be used for the determination of $n_{e}$ profiles and 


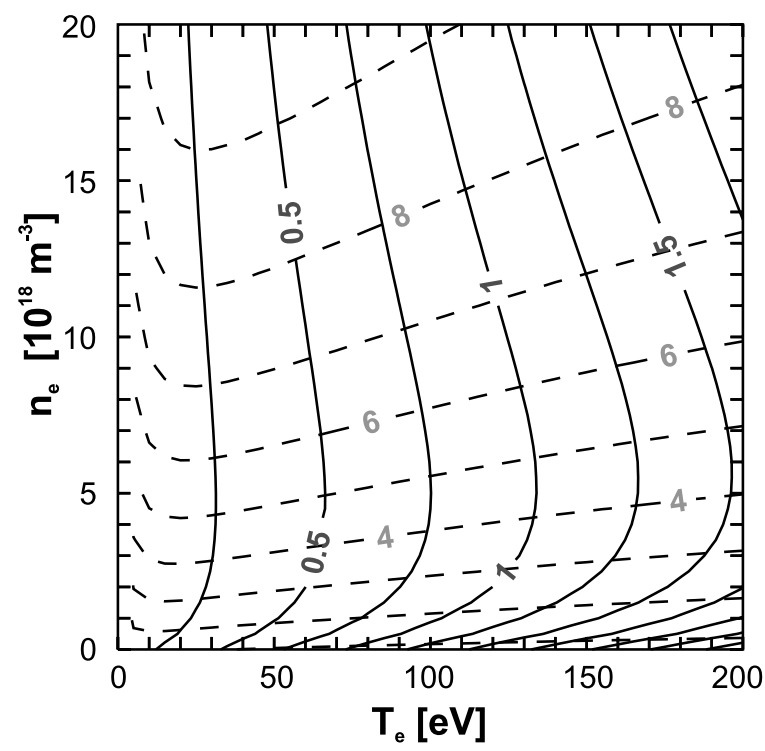

Fig. 13. Calculated line intensity ratios of He for the determination of $n_{e}$ and $T_{e}$. The vertical lines (-) represent the singlet/triplet intensity ratio $I(728 \mathrm{~nm}) /$ $I(706 \mathrm{~nm})$, which is $T_{e}$ dependent, and the horizontal lines (- - -) represent the singlet/singlet intensity ratio $I(668 \mathrm{~nm}) / I(728 \mathrm{~nm})$, which is $n_{e}$ dependent.

also for charge exchange recombination (CXR) spectroscopy. A result ${ }^{18}$ comparing all methods to determine of $n_{e}(r)$ is given in Fig. 11.

The disadvantages - a small penetration depth for thermal atoms and a pulsed operation for the supra- thermal beam-could be overcome by the use of continuous helium beams. Helium can penetrate quite far into the plasma because of its high ionization energy of $24.9 \mathrm{eV}$ (Fig. 12) and is applied for the simultaneous measurement of $n_{e}(r)$ and $T_{e}(r)$ in the plasma edge. ${ }^{29}$ The line intensity ratios of the transitions in the singlet and triplet systems at the wavelengths $\lambda_{1}^{S}=667.8 \mathrm{~nm}$, $\lambda_{2}^{S}=728.1 \mathrm{~nm}$, and $\lambda_{3}^{T}=706.5 \mathrm{~nm}$ are calculated for a local position inside the plasma edge and compared with the values obtained from a collisional-radiative model, ${ }^{20}$ shown in Fig. 13. The profiles can be measured quasicontinuously with a time resolution of $1 \mathrm{~ms}$. Because of the high divergence, this source cannot be used for fluctuation measurements. This would be possible with a supersonic He beam with a beam divergence of $\pm 1 \mathrm{deg}$ now established on TEXTOR (Ref. 19).

The most complex atomic beam systems are neutralized ion beams. They are mainly used to measure impurity densities and temperatures $\left(n_{i m p}, T_{i m p}\right)$ and rotational velocities observing CXR lines. In Fig. 14 the experimental arrangement of a 50-keV $\mathrm{H}$ diagnostic beam at TEXTOR is shown. ${ }^{21}$ This extraction voltage was used because the cross section for the CXR of $\mathrm{H}$ atoms with impurities reaches a maximum value at $\sim 50 \mathrm{keV}$

\section{DIAGNOSTICS WITH LASERS}

In case the excitation by plasma electrons or highly energetic neutrals is not sufficient or not desired, laserinduced fluorescence (LIF) can be used. LIF is the most

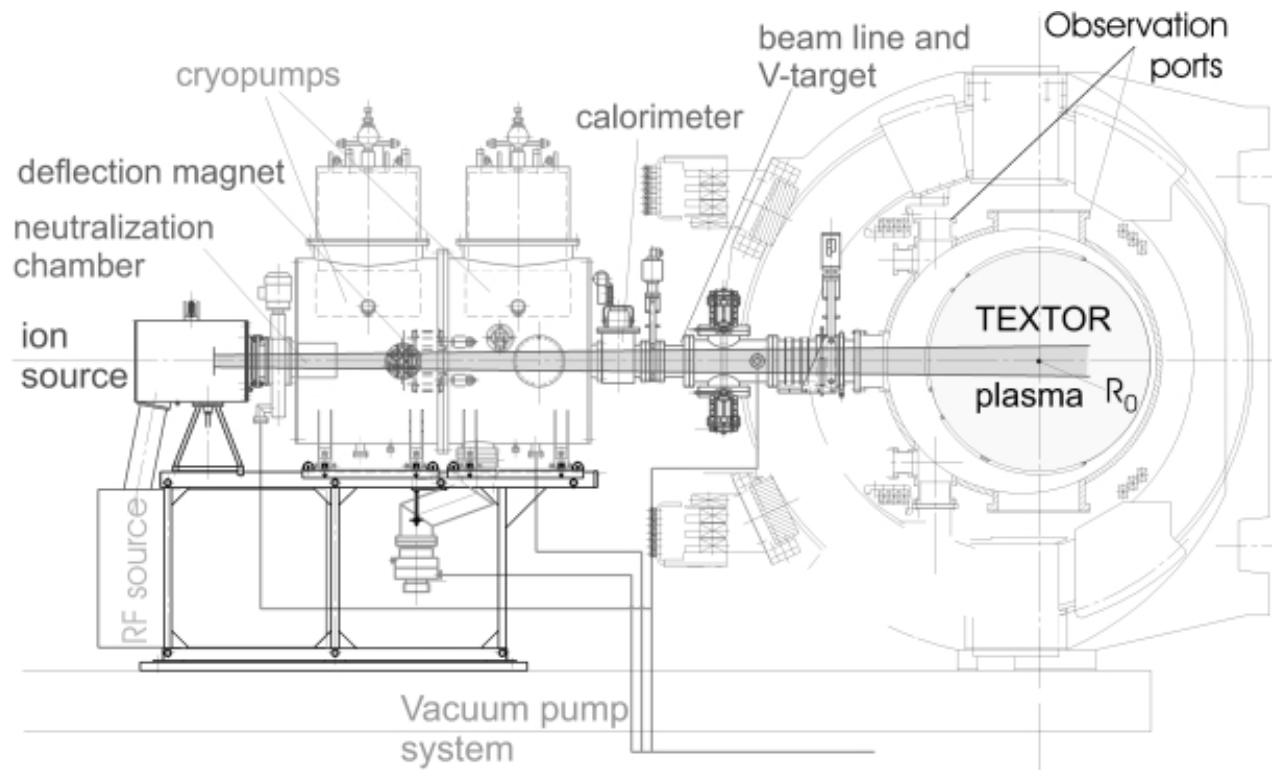

Fig. 14. The 50-keV H diagnostic beam at TEXTOR. 

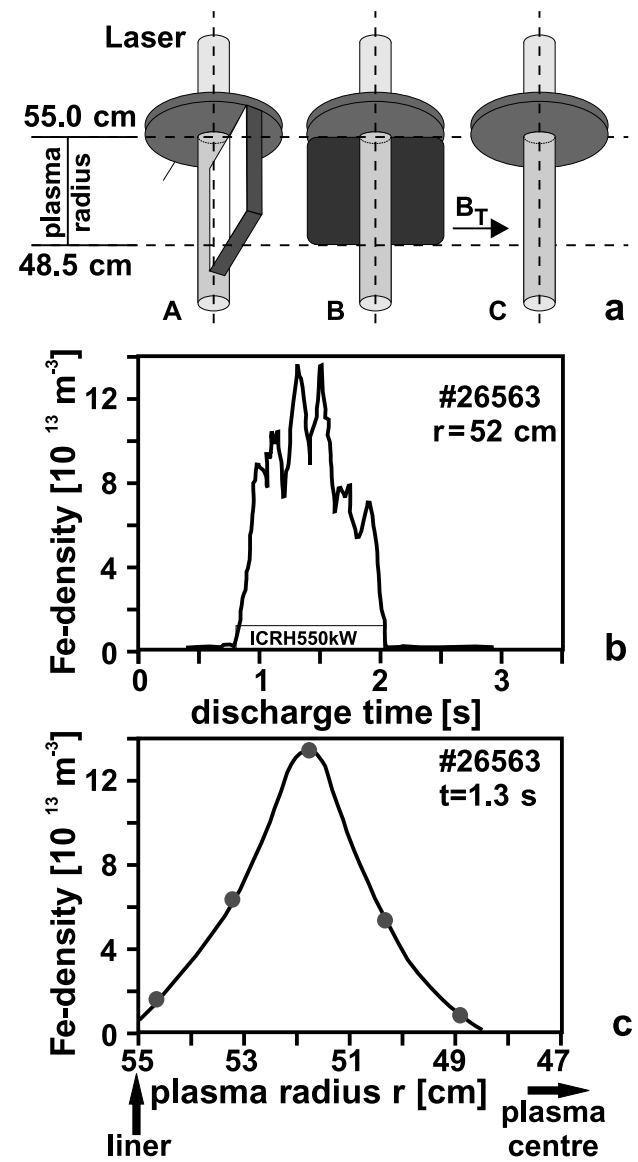

Fig. 15. (a) Arrangement for the measurement of Fe fluxes from a target plate by means of LIF during ICRH in TEXTOR at various positions $\mathrm{A}, \mathrm{B}$, and $\mathrm{C}$ with respect to the magnetic field; (b) Fe densities as a function of time with ICRH, target position $\mathrm{C}$; and (c) radial variations of $\mathrm{Fe}$ densities during ICRH with target position $\mathrm{B}$.

sophisticated-and in the case of light detection and excitation in the vacuum ultraviolet (VUV) range the most difficult-to determine densities and velocity distributions of atoms and ions and has, therefore, unfortunately not been performed very often for the diagnostics of fusion edge plasmas. Nevertheless, the high selective detection sensitivity may enable valuable information to be obtained for the population of excited states. As the laser energy is mostly concentrated in a very narrow wavelength band, a scan over the whole line width for the purpose of Doppler-shift or line-broadening measurements has to be carried out. Commonly, this is accomplished by varying the air pressure in the laser, generating volume that in turn influences the refraction index and thus the emitted laser wavelength. A further advantage of LIF measurements is that knowledge of $T_{e}$ and $n_{e}$ is in most cases not needed.
Measurements can be made even at $n_{e} \approx 0$, i.e., very near the wall. Because of the small dimensions of the exciting laser beam, the spatial resolution can be very good. However, since the resonance lines of the elements, which are at present of highest interest $(\mathrm{H}, \mathrm{D}$, $\mathrm{C}, \mathrm{O}$ ), have their resonance lines in the VUV range, the measurements are rather difficult, especially if many spatial points and spectral profiles are desired. The techniques of how these elements have been diagnosed on TEXTOR with VUV-LIF are described in Ref. 22. However, resonance lines of iron, boron, and silicon lie in a more easily accessible near-ultraviolet range $(\lambda \approx$ $250 \mathrm{~nm}$ ).

Therefore, the first measurements with LIF on ISX-B by Jülich scientists were performed on iron atoms near the wall, where the emission intensities are often too low to be detected. The resonance lines for excitation lie in the near-ultraviolet range, and the laser radiation was produced by a frequency-doubled dye laser. ${ }^{23}$ The method was then applied very successfully on TEXTOR for detecting the strong enhancement of Fe sputtering during ion cyclotron resonance heating ${ }^{24}$ (ICRH) (Fig. 15).

After the introduction of boronization and siliconization of the walls, the interest subsequently turned to the measurement of densities of the lighter elements (B and $\mathrm{Si}$ ), which can still be observed in the near-ultraviolet range. The diagnostic arrangement for fluorescence measurements on TEXTOR is shown in Fig. 16.

Figure 17 shows the raw fluorescence signal versus number of laser shots for a typical plasma discharge. The laser wavelength was continuously scanned over the flattop part of the tokamak discharge. The distribution extends far to the left of the picture, to larger wavelength shifts or higher velocities showing sputtered atoms escaping from the limiter. The second profile comes from particles that are excited by the reflected laser light, so the Doppler shift extends in the other direction. In fact, it is a mirrored image, with smaller amplitude, of the first distribution.

A Thompson distribution can be fitted to the first curve with a half-width of $\sim 5 \mathrm{pm}$, which results in a surface binding energy of $U_{s}=4.9 \mathrm{eV}$. Additionally, the $(1 / e)$ decay length of the fluorescence signals in the plasma gives an estimate for the penetration depth of the sputtered atoms in front of the limiter, which amounts to $\sim 7 \mathrm{~mm}$. From the Doppler shift a velocity of $v=3 \times$ $10^{5} \mathrm{~cm} / \mathrm{s}$ can be deduced.

The high magnetic field of a tokamak like TEXTOR $(\approx 2 \mathrm{~T})$, however, adds an additional complication to such measurements. In Fig. 18 a Si I-line at $251.6 \mathrm{~nm}$ is shown with its splitting into two $\sigma$ components and a central $\pi$ component. Therefore, for absolute total intensity measurements, all these components have to be measured separately and taken into account. For boron such measurements have to be performed only in laboratory experiments ${ }^{25}$ but will also be carried out on TEXTOR in the near future. 


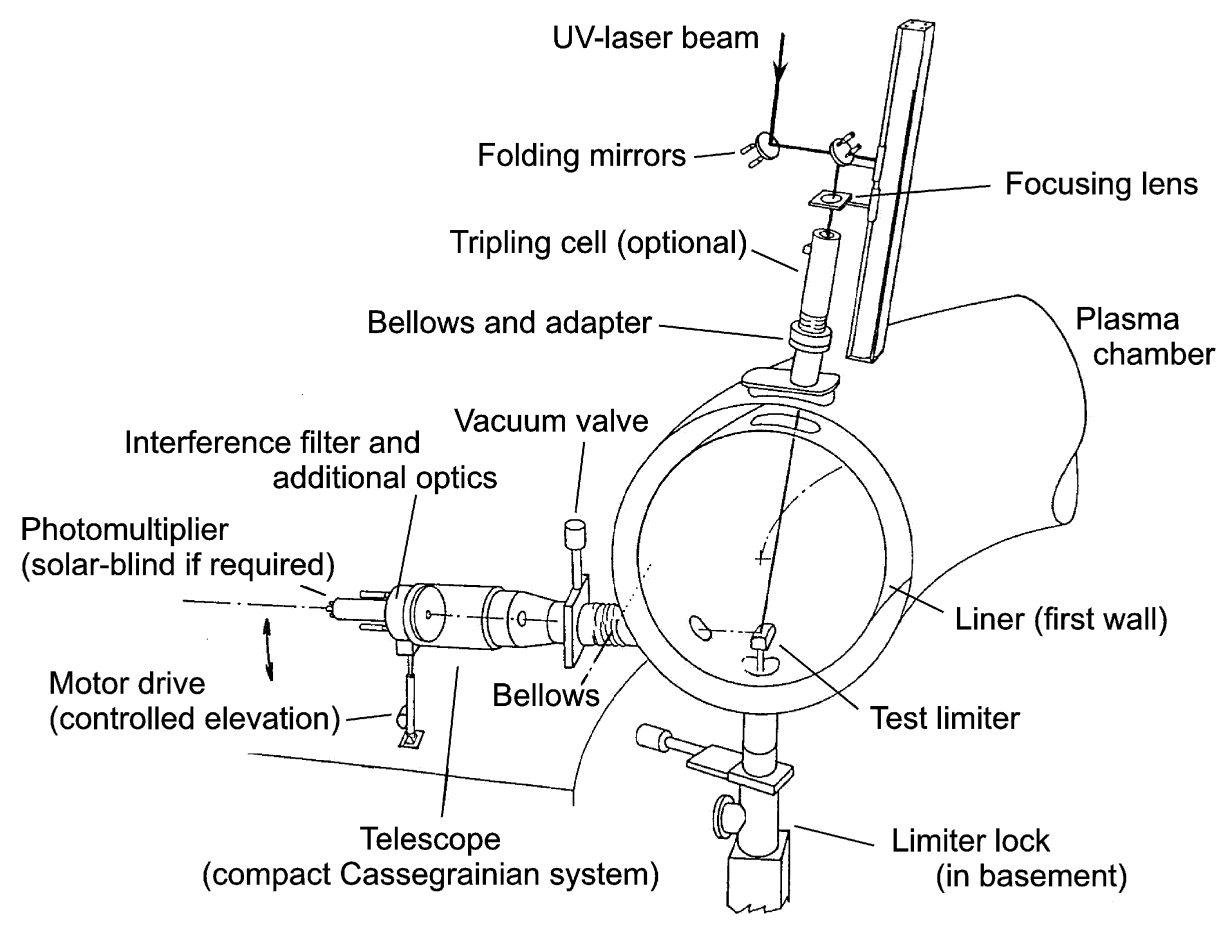

Fig. 16. Experimental arrangement for fluorescence experiments on TEXTOR (Ref. 33).

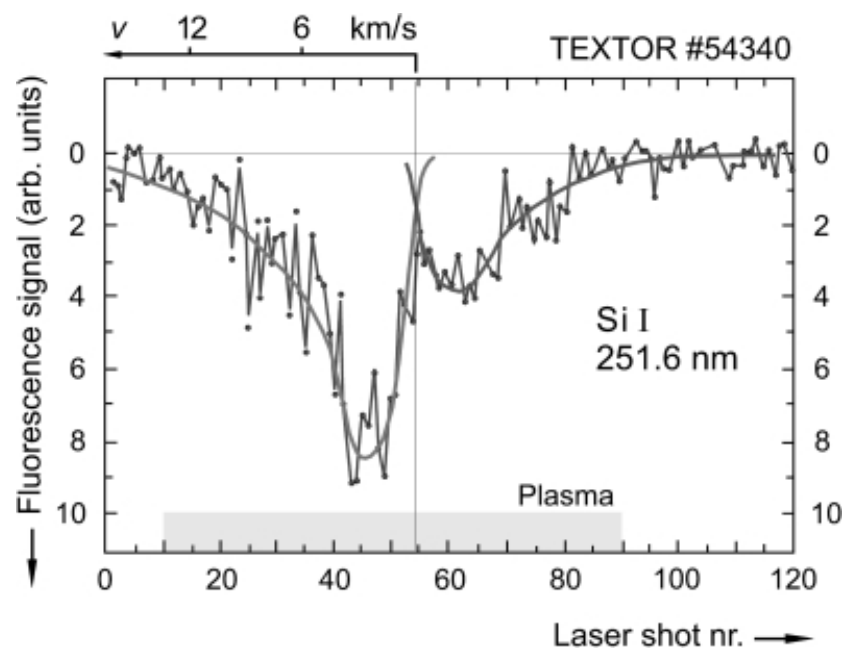

Fig. 17. Fluorescence signal from $\mathrm{Si}$ atoms in front of a silicon carbide test limiter during a plasma discharge. ${ }^{34}$

\section{DIAGNOSTICS WITH PROBES}

Soon after the start of TEXTOR, it has been recognized that the use of electrical probes can be a valuable complement for a full characterization of the plasma edge. Not only do they offer additional parameters, such as plasma potential, flows, density, and most importantly

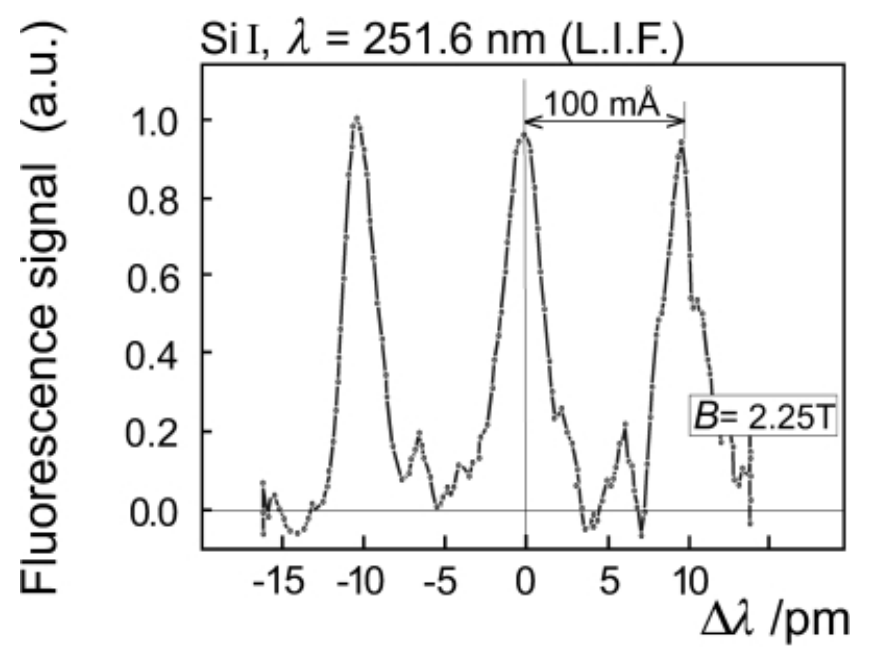

Fig. 18. Zeeman splitting of a Si I-line measured perpendicularly to the toroidal magnetic field in front of a silicon carbide test limiter during a plasma discharge.

their fluctuating part, but also they can be used to compare the different diagnostics, to deliver complementary data, and to check the reliability of the atomic data. This has triggered an extensive research activity, which was enhanced by detailed studies by different research groups of various aspects of probe measurements and schemes. In the following, the three main lines of research activities are sketched. 
A common problem of probes is the understanding of the particle flux parallel to a strong magnetic field, which provides the ion saturation current. Very often a disagreement in the density profiles among different diagnostics has been found. Therefore, a technique has been developed to calibrate density measurements by probes in situ. By using different probe sizes, it has been revealed that a significant current is collected by probe surfaces, which are parallel to the magnetic field. Taking into account a correction factor for the effective collection area of a cuboid good agreement of the measured density compared with that of the Li beam has been obtained. The in situ calibration has been improved by using a rotating cylindrical double probe instead of using different probe cuboids. ${ }^{26}$ Later, it was demonstrated that this probe can also be used to determine the ion temperature. During the rotation of the probe, the saturation currents start to decrease, when the two pins shadow each other. The decrease is caused by the gyro-radius effect of the ions. The ion temperature can then be determined by comparing the screening calculated from measurements with the one obtained from a Monte Carlo simulation. ${ }^{24,27}$

For a long time, probe measurements at TEXTOR have been limited by the accumulated heat flux caused by the fixed position of the probe during the discharge. This has been overcome by constructing pneumatic probe drives, which allowed a deeper penetration, even beyond the last closed flux surface (LCFS), and concomitantly improved the spatial resolution. ${ }^{28}$ Since the exposure of the probe head to the plasma has been shortened, the pin size and probe head could be minimized such that measurements of edge turbulence became feasible. Among the common methods to determine the turbulent particle flux, a new technique has been tested to estimate the electron temperature using the second harmonic of a highfrequency sinusoidal voltage-driven current measurement. The advantage of the method is that the temperature fluctuations can be measured using solely one single tip, which therefore does not have to rely on the local homogeneity of the plasma. ${ }^{29}$

Many experimental studies, e.g., H-mode, need the knowledge about poloidal plasma rotation. However, the diagnostic means are very limited. Already, Hutchinson has developed a model to determine the parallel flow from the ratio of the upstream and downstream saturation currents of two one-sided-shielded probe collectors. ${ }^{30}$ It is obvious that this ratio will change once the angle $\theta$ of the collectors with respect to the magnetic field is varied, which makes the probe sensitive to the perpendicular flow. In order to determine the perpendicular Mach number, the probe model has been extended by taking into account the perpendicular flux into the flux tube and reformulating the Bohm condition at the sheath entrance. ${ }^{31}$ The change of the upstream and downstream ion saturation currents $R$ with inclination angle $\theta$ is obtained from a one-dimensional (1-D) fluid model and has

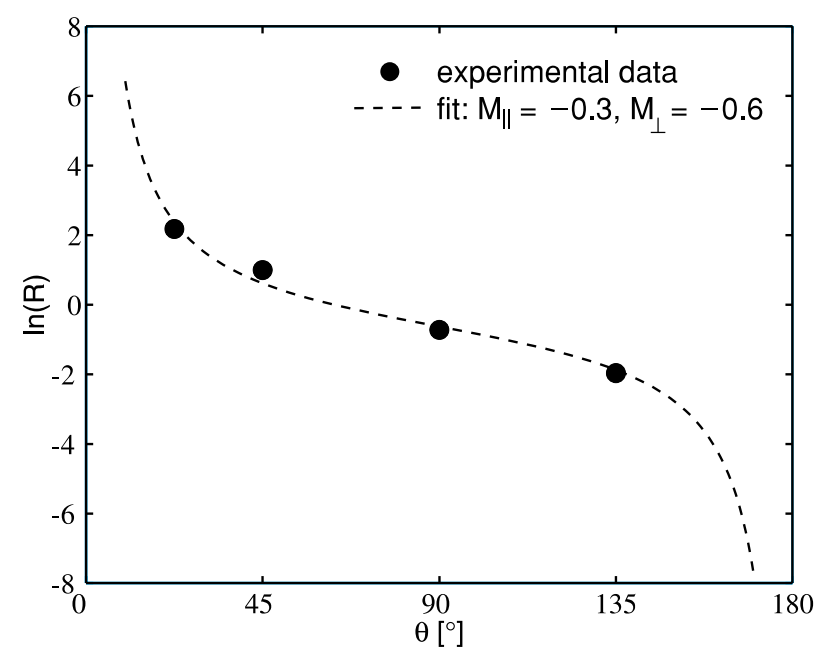

Fig. 19. Upstream and downstream ion saturation current ratio for different probe inclination angles $\theta$. The experimental data are well described by a 1D-fluid model, which gives a best fit for the parallel and perpendicular Mach number.

the form $\ln R=2.21\left(M_{\|}-M_{\perp} \cot \theta\right)$, where $M_{\|}$and $M_{\perp}$ are the parallel and perpendicular Mach numbers.

Figure 19 compares the experimental data with the 1-D fluid model. It can be seen that the data follow well the cotangent dependence on $\theta$. Based on this, the influence of the plasma flow on the floating potential $\phi_{f l}$ could also be studied. It has been found that for a cylindrical tip the contribution of the sheath drop (typically $-3 \mathrm{kT}_{e}$ ) to $\phi_{f l}$ is reduced by a factor of 2 at the most. Furthermore, this effect can be used to determine Mach numbers from the $\phi_{f l}$ of two separated opposite plates. ${ }^{32}$

\section{SUMMARY}

Diagnostics of TEXTOR edge plasmas either by spectroscopic or probe techniques has been an everlasting and challenging task during the past 2 decades. Optical spectroscopy with low and high spatial and spectrally resolving power has contributed considerably to the elucidation of physical and chemical particle release mechanisms from either metallic, carbonized, boronized plasma-facing components or carbon tiles. Especially, in the high-flux, high-temperature regime, these results are unique in fusion community research. Pioneering work has been done on the design and development of various diagnostics (e.g., He beams and VUV-LIF), and many theoretical calculations (e.g., collisional-radiative models for $\mathrm{He}$ and $\mathrm{H}$ ) were performed to interpret the experimental data. Both the work on edge plasma characterization-supported also by probe measurements - and on the release mechanisms of impurities, 
hydrogen, and their molecular compounds have contributed considerably to the knowledge of particle transport. New experiments such as the dynamic-ergodic divertor require higher time and spatial resolution and more sophisticated theoretical models for the interpretation of experimental data.

\section{REFERENCES}

1. A. UNSÖLD, Physik der Sternatmosphären, 2nd ed., Springer, Berlin (1968).

2. P. BOGEN et al., J. Nucl. Mater., 128-129, 157 (1984).

3. A. POSPIESZCZYK, "Diagnostics of Edge Plasmas by Optical Methods," in Atomic and Plasma-Material Interaction Processes In Controlled Thermonuclear, R. V. JANEV and H. W. DRAWIN, Eds., Elsevier, Cadarache (1993).

4. A. POSPIESZCZYK et al., J. Nucl. Mater., 147574 (1987).

5. U. SAMM, Plasma Phys. Control. Fusion, 29, 1321 (1987).

6. S. BREZINSEK, "Untersuchung von atomarem und molekularem Wasserstoff vor einer Graphitoberfläche in einem Hochtemperaturplasma," JÜL-Report 3962, Forschungszentrum Jülich (2002).

7. A. POSPIESZCZYK et al., J. Nucl. Mater., 269, 138 (1999).

8. S. BREZINSEK et al., Contrib. Plasma Phys., 42, 668 (2002).

9. PH. MERTENS et al., Plasma Phys. Control. Fusion, 43, A349 (2001).

10. A. POSPIESZCZYK et al., J. Nucl. Mater., 241-243, 833 (1997).

11. A. POSPIESZCZYK et al., J. Nucl. Mater., 290-293, 947 (2001).

12. A. HUBER, “The 'Video' Program: Spectroscopic Data Display and Analysis for Video Imaging on TEXTOR-94," JÜL-Report 3905, Forschungszentrum Jüelich (2001).

13. A. POSPIESZCZYK et al., J. Nucl. Mater., 162-164, 574 (1989).

14. R. P. SCHORN, "Untersuchungen zur Zerstäubung von Kupfer/ Lithium-Legierungen,” JÜL-Report 2367, Forschungszentrum Jülich (1990).
15. A. POSPIESZCZYK and G. G. ROSS, Rev. Sci. Instrum., 59, 605 (1988).

16. A. POSPIESZCZYK and G. G. ROSS, Rev. Sci. Instrum., 59, 1491 (1988).

17. S. FIEDLER et al., J. Nucl. Mater., 266-269, 1279 (1999).

18. B. SCHWEER et al., J. Nucl. Mater., 196-198, 174 (1992).

19. B. SCHWEER, Trans. Fusion Sci. Technol., 41, 411 (2002).

20. M. BRIX, "Messung von Elektronentemperatur und -dichte mittels Heliumstrahldiagnostik im Randschicht-plasma eines Tokamaks," JÜL-Report 3638, Forschungszentrum Jülich (1999).

21. A. KRETER et al., Proc. 27th Conf. Controlled Fusion and Plasma Physics, Budapest, Hungary, 2000, Europhysics Conf. Abstracts, Vol. 24B, p. 1232 (2000).

22. PH. MERTENS and S. BREZINSEK, "Recycling of Hydrogen Isotopes-From an Identification of Mechanisms in TEXTOR to a Wider Formulation,” Fusion Sci. Technol., 47, 161 (2005).

23. B. SCHWEER et al., J. Nucl. Mater., 93-94, 357 (1980).

24. B. SCHWEER et al., Proc. 13th European Conf. Controlled Fusion and Plasma Heating, Schliersee, Germany, 1986, Europhysics Conf. Abstracts, Vol. III, p. 399 (1986).

25. E. PASCH et al., J. Nucl. Mater., 196-198, 1065 (1992).

26. K. HÖTHKER et al., Rev. Sci. Instrum., 58, 1046 (1987).

27. K. HÖTHKER et al., Rev. Sci. Instrum., 61, 114 (1990).

28. J. BOEDO et al., Rev. Sci. Instrum., 69, 2663 (1998).

29. J. BOEDO et al., Phys. Rev. Lett., 84, 2630 (2000).

30. I. HUTCHINSON, Phys. Fluids, 30, 3777 (1987).

31. H. VAN GOUBERGEN et al., Plasma Phys. Control. Fusion, 41 , L17 (1999).

32. S. JACHMICH et al., Proc. 27th Conf. Controlled Fusion and Plasma Physics, Budapest, Hungary, Europhysics Conf. Abstracts, Vol. 24B, p. 832 (2000).

33. PH. MERTENS and A. POSPIESZCZYK, J. Nucl. Mater., 266269, 884 (1999).

34. PH. MERTENS et al., Proc. 20th Conf. Controlled Fusion and Plasma Physics, Lisboa, Portugal, Europhysics Conf. Abstracts, Vol. 17C, p. 1123 (1993). 